\title{
Proteomic profiling of secretory granules of different $T$ cell subpopulations
}

\author{
H Schmidt*1, C Gelhaus² ${ }^{2}$ M Nebendahl1, M Leippe ${ }^{2}$ and O Janssen ${ }^{1}$
}

Address: ${ }^{2}$ UK-SH Campus Kiel, Institute of Immunology, Molecular Immunology, Kiel, Germany and ${ }^{2}$ Department of Zoophysiology, Zoological Institute, Christian-Albrechts-University, Kiel, Germany

* Corresponding author

from I2th Joint Meeting of the Signal Transduction Society (STS). Signal Transduction: Receptors, Mediators and Genes

Weimar, Germany. 29-3I October 2008

Published: 26 February 2009

Cell Communication and Signaling 2009, 7(Suppl I):A85 doi:I0.1I86/I478-8IIX-7-SI-A85

This abstract is available from: http://www.biosignaling.com/content/7/SI/A85

(c) 2009 Schmidt et al; licensee BioMed Central Ltd.

In cytotoxic T lymphocytes and Natural Killer (NK) cells, effector molecules including granzymes, perforin, granulysin and FasL are stored in specialized granules termed secretory lysosomes (SL). These vesicles represent dualfunctional organelles that obviously combine degradative and exocytotic properties [1]. We previously established an enrichment protocol to define the proteome of SL from NK cells. We found that the protein content of SL very much depends on the function of a given cell type or clone, best reflected by crucial differences in functionally relevant proteins in transformed NK cell lines [2].

In order to compare the lysosomal content of different $\mathrm{T}$ cell subpopulations, we enriched SL from alpha/beta (CD4 or CD8) and gamma/delta (Vdelta 1 or Vdelta2) T cell lines and clones. To this end, the $\mathrm{T}$ cell lysates were separated by density gradient centrifugation on Iodixanol gradients. As described before, for the differential proteome analysis we focused on the fraction that contained most FasL, Lamp1 and Lamp3 (as specific SL or general lysosomal markers) and compared the isolated lysosomal fractions by 2D-DIGE. We found that the protein content of SL of in vitro expanded CD4 and CD8 cells as well as Vdelta1 and Vdelta 2 cells is more similar than for example gamma/delta cells compared to alpha/beta cells. A detailed MALDI-based profile of individual SL proteomes based on more than 1000 picked spots from several DIGE experiments will be presented with a focus on the functionally relevant proteins mentioned above. The observed differences might reveal new aspects of population-spe- cific dynamics of activation/maturation and effector function in the T cell compartment.

\section{Acknowledgements}

Sponsored by the DFG (SFB4I5), the Cluster of Excellence "Inflammation at Interfaces" (to OJ) and the Innovationsfond Schleswig-Holstein (to ML).

\section{References}

I. Lettau M, Schmidt H, et al:: Secretory lysosomes and their cargo in T and NK cells. Immunol Lett 2006, 108:10-19.

2. Schmidt H, et al.: 2-D DIGE analyses of enriched secretory lysosomes reveal heterogeneous profiles of functionally relevant proteins in leukemic and activated human NK cells. Proteomics 2008, 8:291।-2925. 